\title{
Análisis Límite de estructuras de fábrica como problema de contacto unilateral: un enfoque probabilista
}

\section{Limit Analysis of masonry structures as an unilateral contact problem: a probabilistic approach}

\author{
F. Magdalena-Layos $^{(*)(*)}$, J. I. Hernando-García ${ }^{(*)}$
}

\section{RESUMEN}

La evaluación de la seguridad de estructuras antiguas de fábrica es un problema abierto. En los casos en los que no se produce deslizamiento, la aplicación de los teoremas del Análisis Límite Estándar constituye una herramienta formidable por su simplicidad y robustez. Sin embargo, cuando puedan existir mecanismos de inicio de colapso que impliquen deslizamientos, no está asegurada la existencia de una solución única, por lo que es necesaria la búsqueda de otros métodos para tratar la incertidumbre asociada a su multiplicidad. Se propone una simulación por el Método de Monte Carlo, de estructuras antiguas de fábrica, modeladas en forma de problema de contacto unilateral con rozamiento entre cuerpos rígidos. Los resultados servirán de fundamento para futuros análisis por otros métodos probabilísticos menos costosos computacionalmente.

Palabras clave: Estructuras de fábrica; contacto unilateral; rozamiento; Monte Carlo.

\section{ABSTRACT}

Safety assessment of the historic masonry structures is an open problem. In cases where no slip occurs the application of the standard limit analysis theorems constitutes an excellent tool for its simplicity and robustness. However, if the mechanisms of the onset of collapse involve sliding, it is not guaranteed the existence of a single solution, so it is necessary to look for other ways to treat the uncertainty associated with its multiplicity. We propose a simulation by the Monte Carlo Method for ancient masonry structures modeled as an unilateral contact problem between rigid bodies with friction, as a theoretical basis for further analyses by probabilistic methods computationally less expensive.

Keywords: Masonry structures; unilateral contact; friction; Monte Carlo.

(*) Escuela Técnica Superior de Arquitectura - Universidad Politécnica de Madrid (España)

(**) Escuela Técnica Superior de Edificación - Universidad Politécnica de Madrid (España)

Persona de contacto/Corresponding author: fernandomagdalenalayos@gmail.com (F. Magdalena-Layos)

Cómo citar este artículo/Citation: Magdalena-Layos, F., Hernando-García, J. I. (2014). Análisis Límite de estructuras de fábrica como problema de contacto unilateral: un enfoque probabilista. Informes de la Construcción, 66(EXTRA-1): mo15, doi: http://dx.doi. org/10.3989/ic.13.098.

Licencia / License: Salvo indicación contraria, todos los contenidos de la edición electrónica de Informes de la Construcción se distribuyen bajo una licencia de uso y distribución Creative Commons Reconocimiento no Comercial 3.o. España (cc-by-nc). 


\section{INTRODUCCIÓN}

La evaluación de la seguridad de las construcciones históricas de fábrica es un problema difícil debido a la naturaleza del material que las constituye.

Desde comienzos de los años 50 (1) el Análisis Límite ha sido una de las posibles herramientas para su evaluación (2) (3) (4) (5). Se ha propuesto en diferentes formas: considerando la posibilidad de colapso por deslizamiento (6) (7) (8) (9) (10) (11) (12) o no (13) (14), realizado por métodos clásicos (3) (14) o numéricos (4) (5) (7) (8) (9) (10) (11) (12) y considerado como método simplificado (4) o exacto (13).

El estudio propuesto se encuadra entre los que Roca et al. (5), en su reciente revisión de métodos aplicables al estudio de las construcciones históricas de fábrica, clasifican como «Desarrollos informáticos avanzados basados en el Análisis Límite. Análisis de estructuras de bloques» y, más concretamente, dentro de la línea de los que consideran la posibilidad de colapso por deslizamiento y una ley de rozamiento-deslizamiento no asociativa (4) (7) (8) (9) (10) (11) (12). Este último enfoque tiene un interés práctico, dado que, este modo de colapso por deslizamiento puede producirse en aquellas partes de las construcciones históricas sometidas a fuertes empujes según la dirección principal de las juntas y a bajas compresiones según la dirección perpendicular. También tiene un interés teórico, porque incluye, como caso particular, al Análisis Límite Estándar que emplea una ley de rozamientodeslizamiento asociativa, es decir, con ángulos iguales de rozamiento y deslizamiento.

El presente trabajo pretende aportar una vía alternativa de solución a los reparos planteados por algunos autores (9) (11) respecto a la búsqueda de un mínimo absoluto del factor de carga de inicio de colapso, sin salirse de los límites conceptuales del Análisis Límite mediante modelos de bloques rígidos.

El modelo empleado es un conjunto de sólidos rígidos en contacto unilateral, seco y directo, con rozamiento finito y siguiendo una ley de rozamiento-deslizamiento no asociativa. Este modelo trata de representar un material con resistencia a compresión muy superior a las compresiones a las cuales está sometido y escasa o nula resistencia a tracción. No considerar la aportación de la resistencia a tracción del material de la junta está del lado de la seguridad. Además muy a menudo en las construcciones históricas no se dispone de un historial de los daños sufridos a lo largo del tiempo por dicho material y, en algunos casos, ni siquiera se tiene la seguridad de que éste exista en la actualidad.

$\mathrm{Al}$ menos desde los años 80 se han venido formulando este tipo de problemas de contacto unilateral como problemas de complementariedad. Su principal característica es incluir, al menos, una restricción de complementariedad, que se puede expresar como condición de ortogonalidad entre dos vectores positivos, es decir, como producto escalar nulo y se puede escribir [1], siendo en este caso el vector $y$ de variables puramente estáticas y el $z$ de puramente cinemáticas.

$$
y \cdot z=0 ; y \geq 0 ; z \geq 0
$$

Los problemas de este tipo son difíciles (15) (16) (17) y pueden: no tener solución, tener una o tener múltiples solucio- nes. En ellos es fácil comprobar una solución previamente hallada, pero no existe ningún método determinista que garantice encontrar una solución, si ésta existe, o demostrar que no existe en caso contrario. Además, la principal dificultad que plantean desde el punto de vista práctico es (17) «[que muchos programas] son capaces de obtener [sin garantía de éxito] algún tipo de solución ... pero son incapaces de determinar la calidad de la solución obtenida», es decir, saber si está cerca o lejos de la correspondiente al mínimo absoluto. Parece pues necesario un método para calificar las soluciones obtenidas.

Además varios de los autores citados han planteado el reparo de que «Hallar el mínimo [absoluto] factor de carga puede llevar a resultados demasiado conservadores» (11) o que «sólo la menor solución no asociativa garantiza la seguridad ... pero ... existe el peligro de subestimar gravemente la capacidad de la estructura real» (9).

Para intentar resolver estas dudas y obtener un modo de calificación de los resultados, se propone un nuevo método consistente en estudiar todo el rango de las soluciones de inicio de colapso posibles, para analizar, posteriormente, cual es la probabilidad de cada uno de los factores de carga asociados a ellas. Con este nuevo procedimiento se podrá calcular la probabilidad de que el colapso se produzca para un valor menor o igual al de un factor de carga dado.

\section{EVALUACIÓN DE LA SEGURIDAD MEDIANTE PROBABILIDADES}

Dado que no es conocida, a priori, la distribución de frecuencias de los factores de carga de las soluciones, es necesario obtenerla por métodos generales.

Definimos como probabilidad de inicio de colapso, para una acción dada, la relación entre la cantidad de soluciones para las que el colapso se inicia con una acción menor o igual que la dada y la cantidad total de soluciones de inicio de colapso. Las cantidades, que de modo general llamamos volúmenes, pueden obtenerse por métodos exactos o aproximados.

Desde este enfoque, la solución del problema tiene dos fases principales: obtención del valor del factor de carga para las soluciones posibles y cálculo de los volúmenes que engloban soluciones con factor de carga menor o igual que uno dado.

\subsection{Desarrollo y comparación del cálculo por los métodos exacto y de Monte Carlo}

Los métodos exactos (18) sólo son aplicables a pequeños problemas, por tanto, en primer lugar se va a mostrar, sobre el siguiente ejemplo elemental, el desarrollo y la equivalencia del método aproximado de Monte Carlo y el exacto.

Se propone modelar una junta continua, parte inferior izquierda del cuerpo de la Figura 1.1, mediante su discretización en múltiples apoyos. Cuando el contacto es perfecto (Figura 1.2) éste se produce en todos los apoyos y la llamaremos junta perfecta. Cuando es imperfecto sólo se producirá en algunos, llegando, en el caso de máxima imperfección de la junta (Figura 1.3), a producirse en un único apoyo, a ésta la denominaremos junta extremadamente imperfecta. Todos los casos posibles se encuentran entre los extremos. 

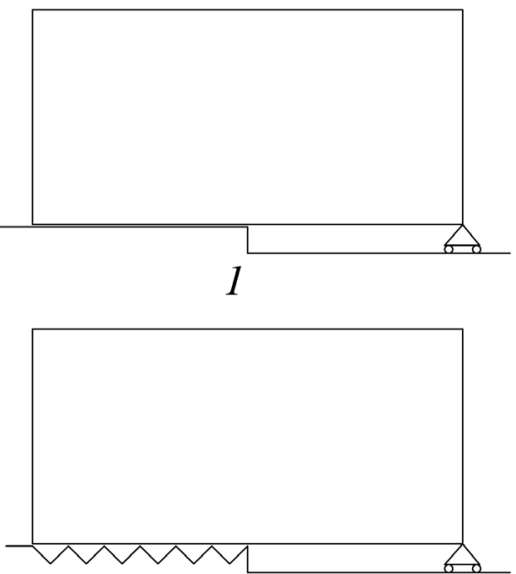

2

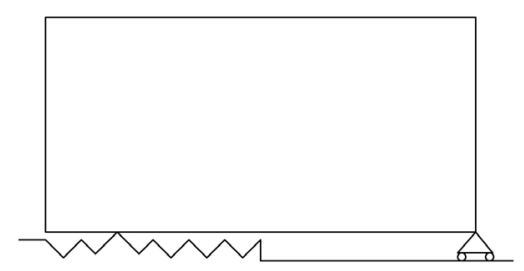

3

Figura 1. Discretización de una junta continua (1) mediante múltiples puntos de contacto (2 y 3 ).

Se pretende obtener la totalidad de las soluciones de inicio de colapso posibles, correspondientes a un mecanismo determinado, con el fin de estudiar la distribución de los valores de las acciones desestabilizantes correspondientes.

De modo muy sintético, una solución de inicio de colapso debe ser una solución estática válida, que representamos como $y \in\left\{E_{y}\right\}$, y debe ser una solución cinemática válida, que representamos como $z \in\left\{K_{z}\right\}$.

Además deben cumplirse las condiciones de contacto unilateral entre ambas, que expresan la imposibilidad de que se inicie un desplazamiento elemental, en un punto de contacto, a menos que el estado de solicitaciones en éste haya alcanzado su valor límite. Estas últimas condiciones, puesto que las soluciones estática y cinemática se han formulado en función de dos vectores positivos $y, z$, pueden escribirse como producto escalar nulo $y \cdot z=0$. Por tanto, una solución de inicio de colapso es la que cumple las restricciones [2].

$$
y \in\left\{E_{y}\right\} ; y \cdot z=0 ; z \in\left\{K_{z}\right\}
$$

Las especiales características de las restricciones que gobiernan el problema [1] hacen que, una vez elegida de algún modo una parte de la solución y sustituida en el problema original, éste se convierta en lineal, quedando reducido a un sistema de ecuaciones e inecuaciones lineales. Eligiendo un mecanismo de inicio de colapso $z=z$, el problema queda reducido a un problema lineal con variables estáticas [3]

$$
z=z^{\prime} \quad y \in\left\{\mathrm{E}_{y}\right\} ; y \cdot z^{\prime}=0
$$

En el primer ejemplo, un cuerpo, apoyado en el lado derecho sobre un apoyo deslizante y en el izquierdo sobre una junta de contacto con rozamiento, se somete a la acción desestabilizante de una fuerza externa horizontal $f$ que provocará su fallo por deslizamiento hacia la derecha (Figura 2).

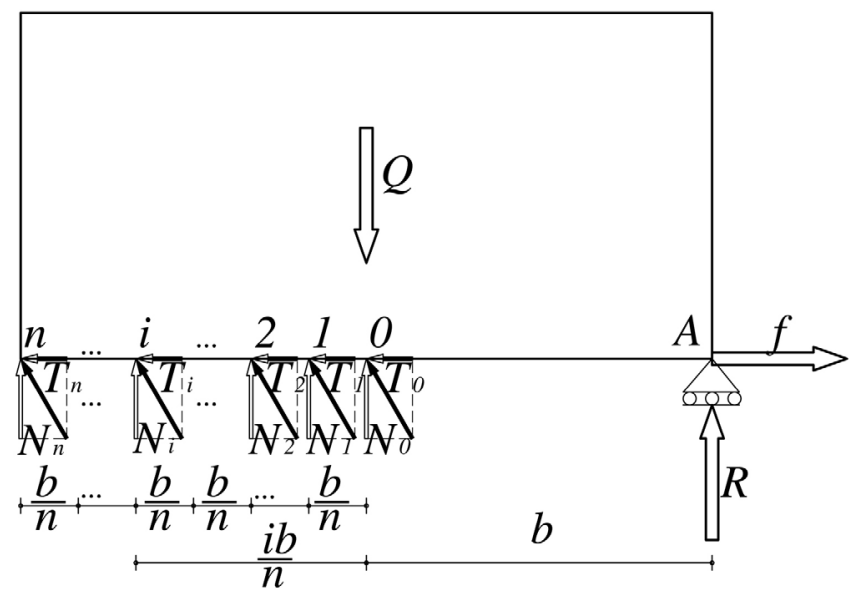

Figura 2. Diagrama de sólido libre (de fuerzas) para una solución estática extrema correspondiente a un mecanismo de deslizamiento hacia la derecha.

El ejemplo se ha elegido por ser uno de los más simples posibles, lo cual permite mostrar los resultados gráficamente.

Se formula el mecanismo de colapso $z=z^{\prime}$ consistente en el deslizamiento hacia la derecha, por medio de sus restricciones estáticas equivalentes, haciendo que la relación entre tangencial y normal alcance en todos los puntos de contacto el valor del coeficiente de rozamiento del material.

Expresado en forma clásica como un problema de estática, discretizada la junta del modo descrito y planteadas las ecuaciones de equilibrio para el caso de $n+1$ apoyos (Figura 2), se comprueba que incluso para $n=1$ el problema está indeterminado.

Sustituyendo los valores límites de las restricciones de cedencia en las ecuaciones de equilibrio, se pueden escribir las ecuaciones estáticas del problema, quedando éste reducido a un sistema de igualdades y desigualdades lineales.

La función a evaluar $f$, empleando la ecuación de equilibrio de fuerzas horizontales, se puede escribir [4], llamando $\phi$ a la $\operatorname{tag} \varphi$ y siendo $\varphi$ el ángulo de rozamiento (estático y en seco):

$$
f=\phi\left(Q-\sum_{i=1}^{n} \frac{i}{n} N_{i}\right)
$$

El conjunto de soluciones posibles, utilizando las ecuaciones de equilibrio de fuerzas verticales y de momentos, quedan delimitadas por las desigualdades [5], debiendo cumplirse la última "para todos los valores de $i$ de o a $n$ ».

$$
\begin{aligned}
& \sum_{i=1}^{n} \frac{i}{n} N_{i} \geq 0 \\
& Q-\sum_{i=1}^{n}\left(1+\frac{i}{n}\right) N_{i} \geq 0 \\
& N_{i} \geq 0 \quad \forall i: i=0 . . n
\end{aligned}
$$

Cada desigualdad divide el espacio en dos semiespacios, en uno de los cuales se cumple la desigualdad y en el otro, no. El conjunto de todas las desigualdades define un cuerpo poliédrico convexo, en el interior del cual se cumplen todas ellas y que, por tanto, contiene todas las soluciones de inicio de colapso para el mecanismo en estudio. Si este poliedro convexo es además cerrado recibe el nombre de politopo. 


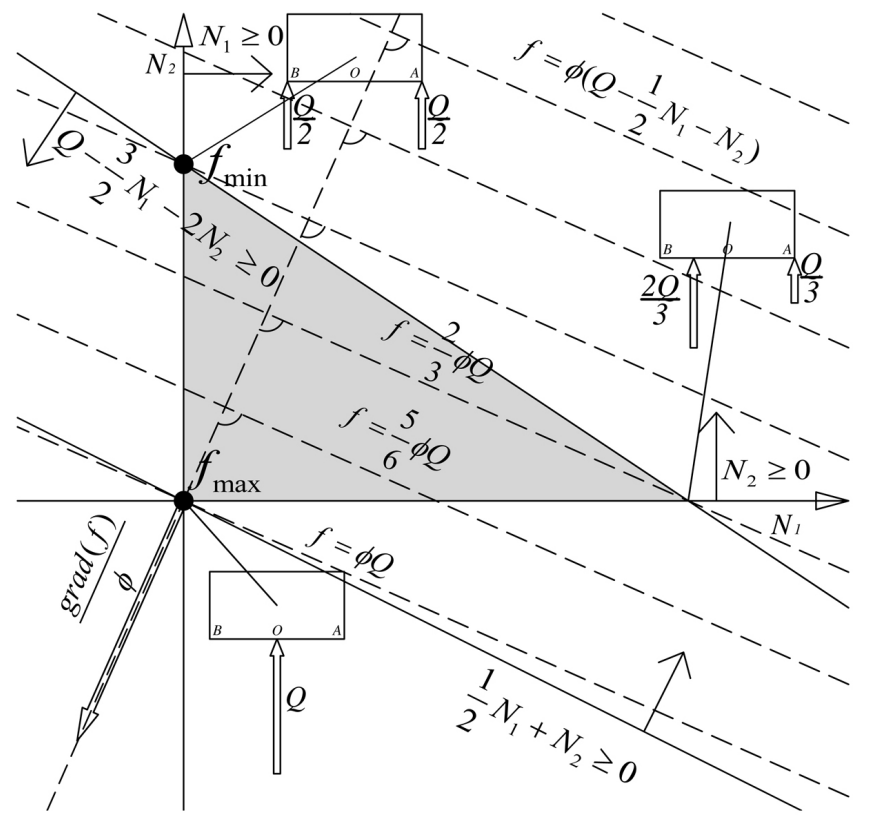

Figura 3. Conjunto de soluciones de inicio de colapso para el caso $\mathrm{n}=2$.

En el caso en que $n=2$, es decir, tres apoyos con rozamiento en la izquierda y uno deslizante en la derecha, el problema aún puede representarse gráficamente. Esto permite mostrar la distribución de los valores de la fuerza desestabilizante $f$ (Figura 3), adoptando la forma de triángulo, que es el politopo más simple en 2 dimensiones. En la figura también se representan, referidas a los vértices del triángulo, las reacciones verticales sobre la junta y el apoyo deslizante correspondientes a las soluciones de los tres casos extremos, en que únicamente una de las reacciones sobre los apoyos con rozamiento no es nula.

En la Figura 4, siendo $\cap$ el símbolo de intersección, se representa el politopo correspondiente a las soluciones en que el colapso se inicia con una acción menor o igual que $f=5 \phi Q / 6$

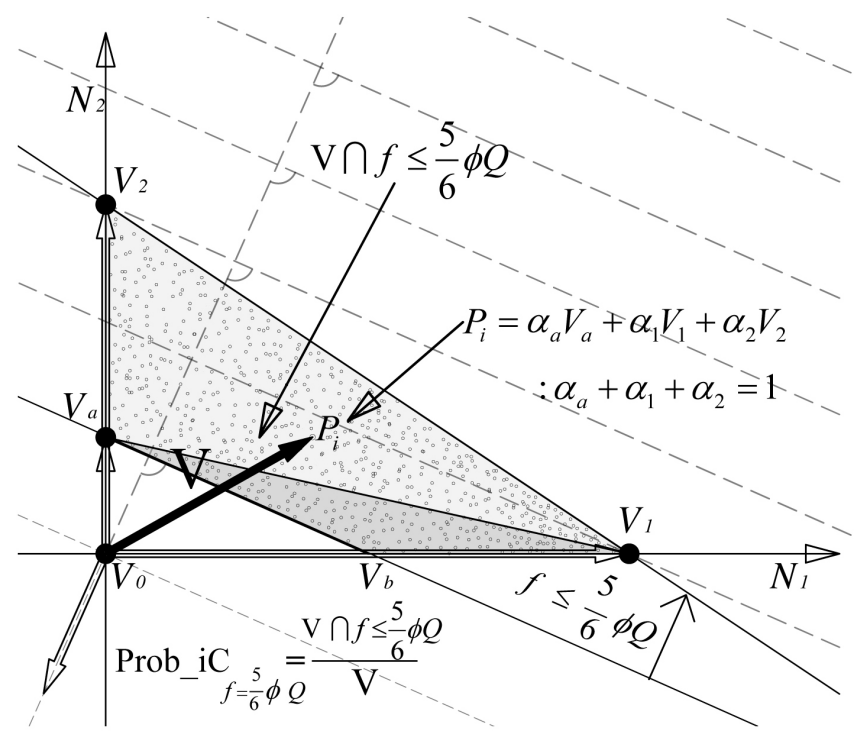

Figura 4. Procedimientos de obtención del volumen de un politopo por triangulación y de un muestreo uniforme sobre el mismo politopo. y su descomposición en triángulos. Los volúmenes pueden obtenerse por métodos exactos (18), en este caso por triangulación y en el $n$-dimensional por descomposición en simplex, que son la generalización de un triángulo a $n$ dimensiones. Así se ha realizado, empleando el software VINCI (19) para computación exacta del volumen de un politopo.

Sin embargo, el uso de estos métodos exactos está restringido a casos con un pequeño número de dimensiones.

También pueden obtenerse los volúmenes por el método de Monte Carlo, siendo especialmente apropiado en los casos de muchas dimensiones. Realizado (para el caso en que $n=10$ ), utilizando el procedimiento que se describirá a continuación, se han comparado los resultados con los obtenidos por el método exacto, comprobando que, a efectos prácticos, son indistinguibles.

\subsection{Simplificación del cálculo por el método de Monte Carlo}

Una vez comprobada la equivalencia de ambos métodos, se continua desarrollando el método de Monte Carlo. A diferencia del método exacto es posible evitar el paso de calcular los volúmenes para todos los factores de carga en estudio, ya que, si se dispone de un algoritmo que permita un muestreo aleatorio y uniforme de los puntos que representan las distintas soluciones de inicio de colapso posibles, se puede realizar un muestreo directo de los valores de las acciones desestabilizantes $f$ obteniendo como resultado su distribución de frecuencias. En el caso de acciones desestabilizantes múltiples se haría con los factores de carga.

$\mathrm{Al}$ igual que en el procedimiento exacto, el primer paso consiste en hallar los vértices del politopo. En el presente trabajo esto se hace de modo determinista. A partir de ellos se obtendrán los puntos del politopo que constituirán la muestra, mediante el procedimiento que se describe a continuación.

El método más clásico de muestreo por Monte Carlo, conocido como método de rechazo, no es viable, dado que en el caso de comprobación estudiado con $n=10$ se admitiría un resultado por cada más de tres millones probados. Por ello se implementa un procedimiento específico (20) que permite realizar un muestreo uniforme de los puntos de un politopo (Figura 4).

Aplicando dicho procedimiento, en el caso de junta perfecta y para diferentes valores de $n$, se comprueba mediante las gráficas de frecuencias (Figura 5.1) y de frecuencias acumuladas (Figura 5.2) que conforme aumenta el número de puntos de contacto disminuye la varianza de los valores de la acción desestabilizante de la muestra obtenida.

Del mismo modo se hallan las distribuciones, para $n=100$, de frecuencias de los puntos de paso de la resultante (Figura 6 izquierda) y de los valores de la acción desestabilizante (Figura 6 derecha). En ambos casos, la curva señalada con 2 corresponde al caso de junta perfecta y la señalada con 3 al de extremadamente imperfecta. Obsérvese que la curva $n=100$ de la Figura 5.1 es la misma que la curva 2 de la Figura 6 derecha.

Es posible otra simplificación en el procedimiento. Se puede comprobar que en el caso de junta extremadamente imper- 

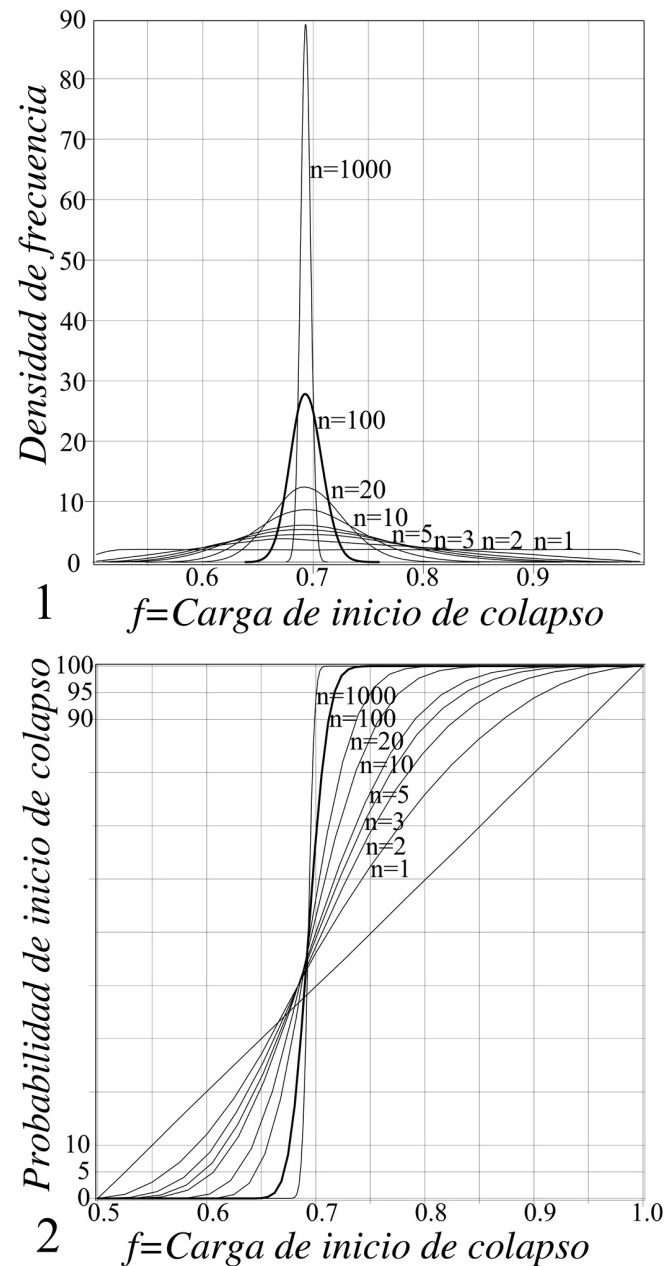

Figura 5. Funciones de distribución (1) y acumulada de frecuencias

(2), de la acción desestabilizante, para distintos valores de n.

fecta las soluciones coinciden con los vértices del politopo y éstos a su vez coinciden con los casos en que hay contacto en un único punto de cada cara de contacto. Aprovechando este hecho, se pueden obtener directamente las soluciones extremas, es decir, los vértices del poliedro de soluciones de inicio de colapso, tal y como se va a hacer en los siguientes ejemplos.

\subsection{Aplicación a varios cuerpos en contacto}

Para mostrar la implementación de esta nueva simplificación, en el siguiente ejemplo se estudian las relaciones de contacto entre tres bloques, en concreto la resistencia a la separación horizontal (a «tracción») de los dos inferiores, sometidos a las solicitaciones transmitidas por el peso del bloque superior que actúan en sentido perpendicular a la acción desestabilizante. A este comportamiento, que puede representar razonablemente el efecto de la traba, es al que Rankine denominó «tenacidad friccional».

Es necesario comprobar, con carácter previo, en cuantas de estas caras de contacto se pueden elegir aleatoriamente los puntos de paso de su resultante. Como se observa (Figura 7.3) una vez elegido el punto de paso y el valor de la resultante en la cara superior, quedan determinados ambos en la cara inferior. De ello se deduce que el muestreo puede realizarse, exclusivamente, sobre las dos caras de contacto con el bloque superior (Figura 7.2).

Para que pueda existir equilibrio en el bloque superior es necesario que al menos uno de los $N_{L j}$ y uno de los $N_{R k}$ (Figura 7.1) sean estrictamente positivos. Los puntos extremos del politopo (soluciones extremas) serán aquellos en los que sólo uno de los $N_{L j}$ y uno de los $N_{R k}$ sean estrictamente positivos (Figura 7.2). Se pueden plantear directamente todas las combinaciones posibles, siendo la condición, para el caso en que el contacto se produce en el punto $j$ de la junta izquierda y $k$ de la junta derecha [6]. El número total de estas combinaciones es $(n+1)^{2}$.

$$
\begin{aligned}
& N_{L j}>0 ; N_{L i}=0 \forall i \neq j \\
& N_{R k}>0 ; N_{R i}=0 \forall i \neq k
\end{aligned}
$$

Como parte de los resultados del método propuesto se obtiene el mecanismo de inicio de colapso que corresponde a cada una de las soluciones. El presente ejemplo tiene tres posibles mecanismos de inicio de colapso por deslizamiento puro: deslizando el bloque inferior izquierdo, el inferior derecho o ambos a la vez.

Los resultados muestran que las soluciones de inicio de colapso que corresponden a cada mecanismo no son igualmente probables. En el caso de junta perfecta no se obtiene ningún caso de deslizamiento simultáneo, siendo la frecuencia sólo a derechas o sólo a izquierdas del 50 \% para cada una. En el caso de junta extremadamente imperfecta, con $n$ puntos de contacto, las soluciones deslizando sólo a la izquierda o sólo a la derecha son $n / 2$ veces más frecuentes, cada una de ellas, que las de deslizamiento simultáneo.

Para completar la exposición, se analiza otro modelo muy sencillo, estudiado por Fishwick (8), en el cual se han fijado las dimensiones para que las restricciones relativas al deslizamiento no sean las únicas que pueden alcanzar sus valores límites (Figura 8 izquierda).

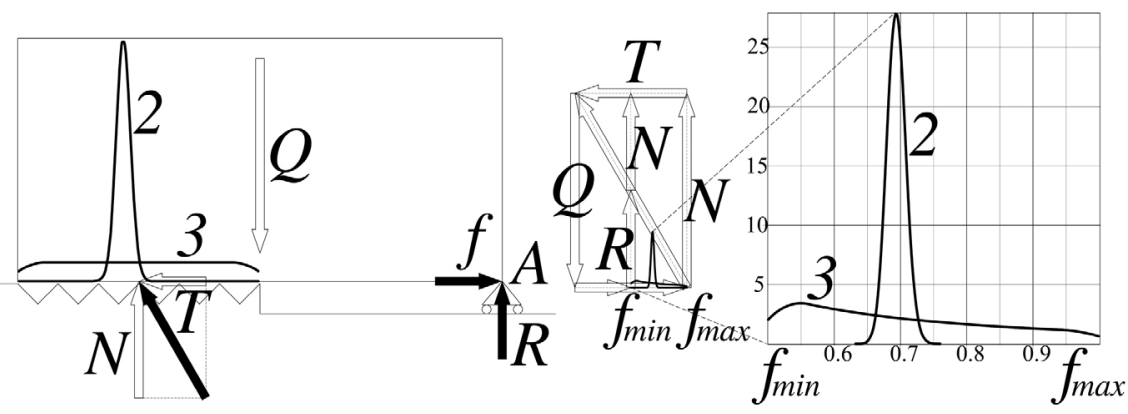

Figura 6. Distribuciones de frecuencias de las acciones desestabilizantes (derecha) y de los puntos de paso de la reacción de contacto (izquierda). 


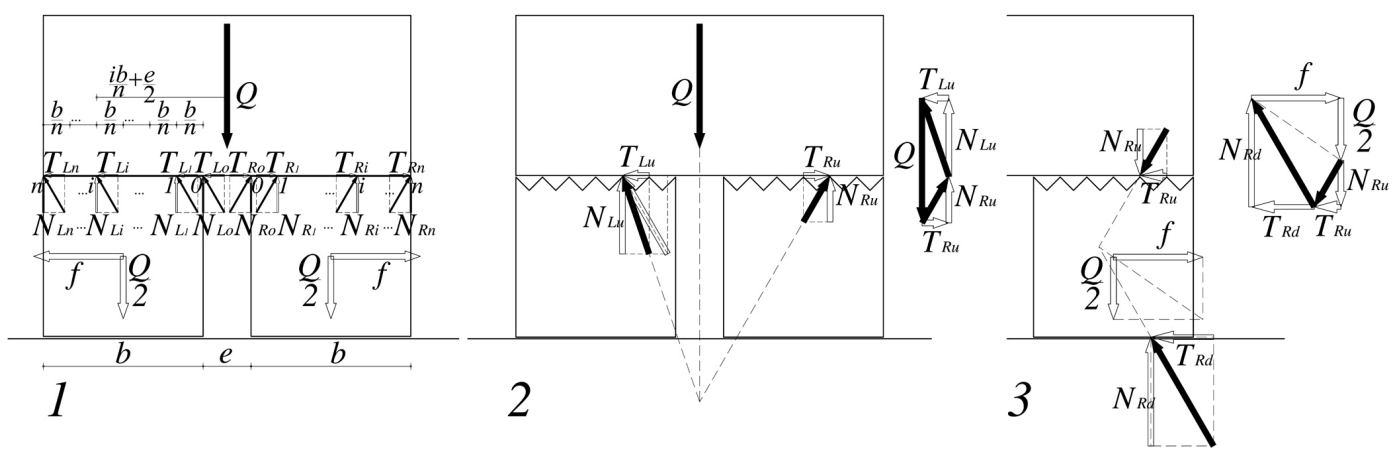

Figura 7. Diagramas de sólido libre (de fuerzas) para las soluciones estáticas extremas correspondientes a los tres mecanismos.

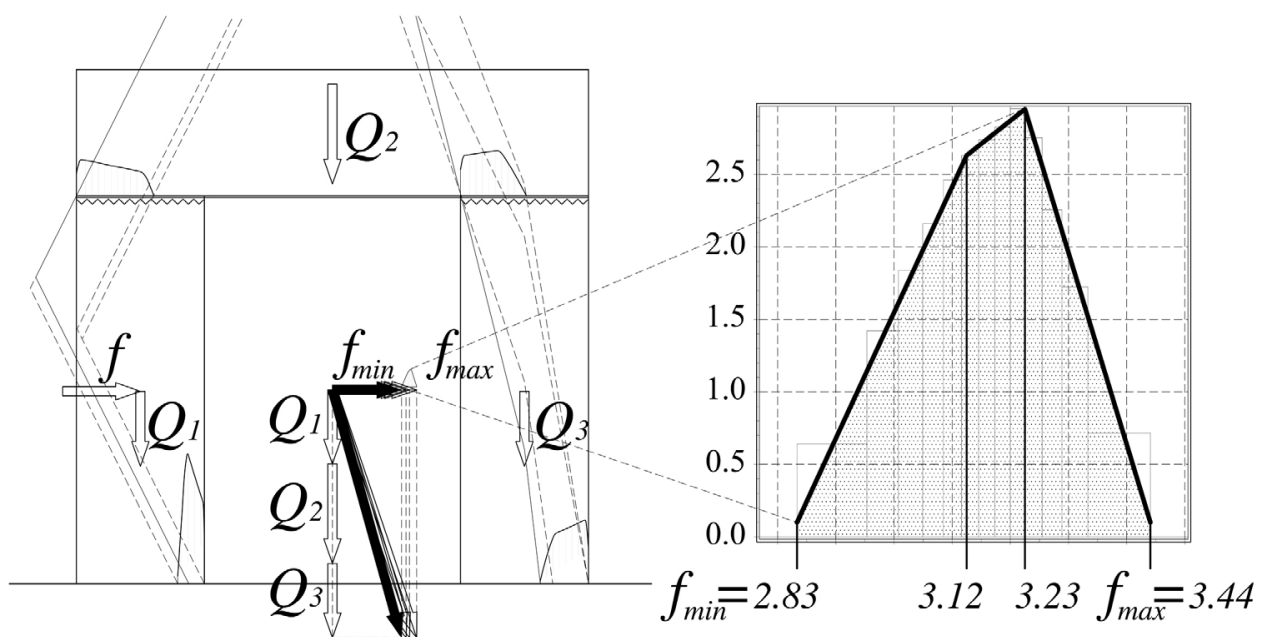

Figura 8. Funciones de distribución de los puntos de paso y de la acción desestabilizante, para el caso de juntas extremadamente imperfectas.

Restringiendo el análisis al mecanismo por deslizamiento del bloque izquierdo, único válido de inicio de colapso, debe completarse el procedimiento ya expuesto con una posterior etapa de rechazo de aquellas soluciones que incumplen la restricción de fallo por vuelco.

En la Figura 8 se representan los resultados para el caso de juntas extremadamente imperfectas, que es el caso más desfavorable en todos los ejemplos. En el lado izquierdo se representan las distribuciones de los puntos de paso de las resultantes, existiendo zonas de las caras de contacto por las cuales no pueden pasar las resultantes pues violarían la restricción de vuelco. En el lado derecho se representa la distribución de los valores de la acción desestabilizante $f$. Los valores máximo y mínimo coinciden, respectivamente, con los que se obtienen aplicando el Análisis Límite Estándar y el procedimiento enumerativo de Fishwick (8). Puede observarse que ambos valores extremos son extremadamente improbables.

En la Figura 9 se resumen, para el caso de junta extremadamente imperfecta, los resultados obtenidos con distintas agrupaciones de dos o tres bloques.

\section{CONCLUSIONES}

Para los casos estudiados, queda demostrado que no es posible determinar a priori la distribución de frecuencias de los valores de las acciones desestabilizantes y que los valores extremos son muy infrecuentes en comparación con la gran cantidad de casos intermedios.
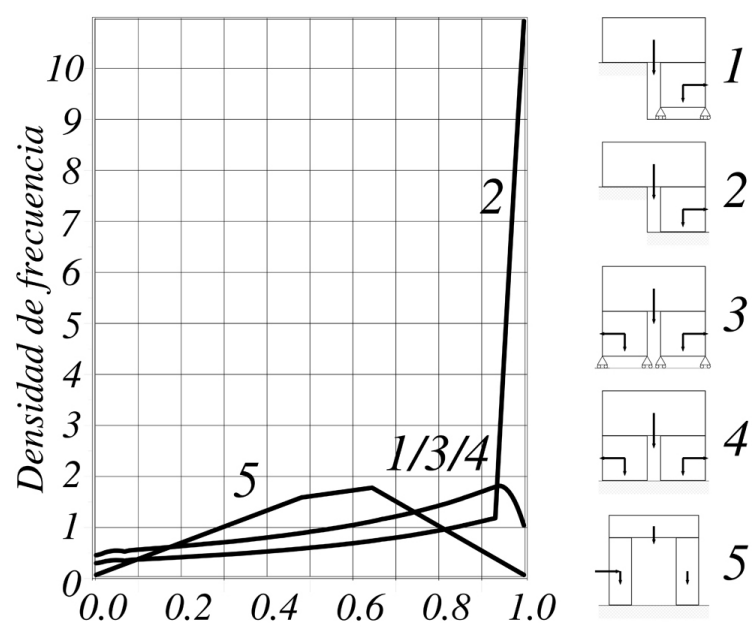

5

Figura 9. Comparación de las distribuciones de frecuencias para el caso de juntas extremadamente imperfectas sobre los distintos modelos.

Estos nuevos resultados concuerdan con lo conjeturado por los autores citados (9) (11) y confirman el interés de profundizar en el desarrollo del nuevo método propuesto. Éste debe entenderse a nivel de investigación básica, su objetivo es obtener datos a partir de los cuales verificar o refutar las hipótesis planteadas e implementar otros métodos probabilistas menos costosos computacionalmente que el método de Monte Carlo. 

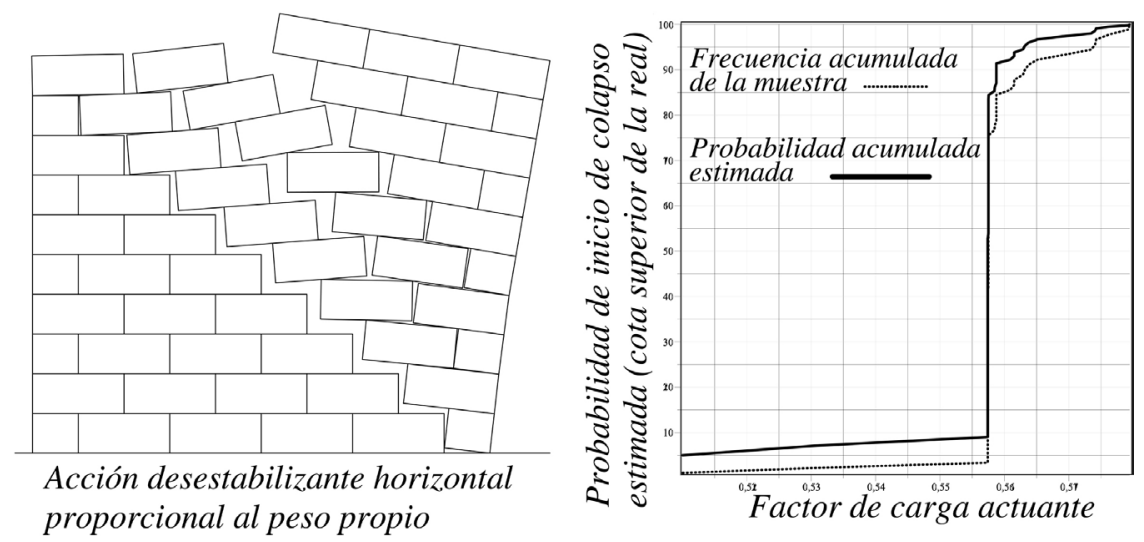

Figura 10. Ejemplo de aplicación de otros métodos probabilistas menos exactos pero menos costosos computacionalmente.

Uno de estos métodos menos costosos, que adopta, para la probabilidad de inicio de colapso, un enfoque de cota superior (Figura 10) y, por tanto, seguro, se ha desarrollado recientemente (10).

\section{DESARROLLOS}

El método propuesto, en su implementación actual, está limitado a ejemplos con pequeño número de caras en contacto. Esto se debe, tanto a la obtención determinista de todas las posibles soluciones extremas, como al creciente número de mecanismos de inicio de colapso posibles según aumenta el número de cuerpos en contacto.

La solución al primer problema se está desarrollando mediante la obtención probabilista de las soluciones extremas. Para el segundo, se está trabajando en métodos de muestreo que cubran uniformemente el espacio de posibles soluciones y aplicables a casos de mayor tamaño.

\section{REFERENCIAS}

(1) Kooharian, A. (1952). Limit analysis of voussoir (segmental) and concrete arches. Proceedings of American Concrete Institute, 49(12): 317-328.

(2) Gilbert, M. (2007). Limit analysis applied to masonry arch bridges: state-of-the-art and recent developments. In 5 th International Arch Bridges Conference (pp. 13-28).

(3) Huerta, S. (2005). Mecánica de las bóvedas de fábrica: el enfoque del equilibrio. Informes de la Construcción, 56(496): 73-89, doi: http://dx.doi.org/10.3989/ic.2005.v57.i496.496.

(4) Orduña, A., Lourenço, P.B. (2001). Limit analysis as a tool for the simplified assessment of ancient masonry structures. En Historical Constructions, (pp. 511-520). Guimarães: University of Minho.

(5) Roca, P., Cervera, M., Gariup, G. (2010). Structural analysis of masonry historical constructions. Classical and advanced approaches. Archives of Computational Methods in Engineering, 17(3): 299-325, doi: http://dx.doi.org/10.1007/ s11831-010-9046-1.

(6) D'Ayala, D. F., Tomasoni, E. (2008). The structural behaviour of masonry vaults: Limit state analysis with finite friction. En Structural analysis of historic construction, (pp. 3-19). doi: http://dx.doi.org/10.1201/9781439828229.

(7) Ferris, M. C., Tin-Loi, F. (2001). Limit analysis of frictional block assemblies as a mathematical program with complementarity constraints. International Journal of Mechanical Sciences, 43(1): 209-224, doi: http://dx.doi.org/10.1016/ Soo20-7403(99)00111-3.

(8) Fishwick, R.J. (1996). Limit analysis of rigid block structures (Tesis doctoral). Portsmouth: Department of Civil Engineering-University of Portsmouth. http://ethos.bl.uk/OrderDetails.do?uin=uk.bl.ethos.310412.

(9) Gilbert, M., Casapulla, C., Ahmed, H. M. (2006). Limit analysis of masonry block structures with non-associative frictional joints using linear programming. Computers \& Structures, 84(13-14): 873-887, doi: http://dx.doi.org/10.1016/j. compstruc.2006.02.005.

(10) Magdalena, F. (2013). El problema del rozamiento en el análisis de estructuras de fábrica mediante modelos de sólidos rígidos (Tesis doctoral). Madrid: Universidad Politécnica de Madrid.

(11) Orduña, A., Lourenço, P. B. (2005). Three-dimensional limit analysis of rigid blocks assemblages. Part II: Load-path following solution procedure and validation. International journal of solids and structures, 42(18): 5161-5180, doi: http://dx.doi.org/10.1016/j.ijsolstr.2005.02.011.

(12) Tran-Cao, T.R.I. (2009). Collapse analysis of block structures in frictional contact (Tesis doctoral). Sydney: The University of New South Wales.

(13) Heyman, J. (2008). The plasticity of unreinforced concrete. Magazine of Concrete Research, 6o(8): 555-559, doi: http:// dx.doi.org/10.1680/macr.2008.60.8.555.

(14) Ochsendorf, J. A. (2002). Collapse of masonry structures (Tesis doctoral). Cambridge: University of Cambridge.

(15) Cottle, R.W., Pang, J.S., Stone, R.E. (2009). The linear complementarity problem. SIAM Classics in Applied Mathematics, doi: http://dx.doi.org/10.1137/1.978089871900o. 
(16) Garey, M. R., Johnson, D. S. (1979). Computers and intractability. New York: Freeman.

(17) Hu, J., Mitchell, J. E., Pang, J.S., Bennett, K. P., Kunapuli, G. (2008). On the global solution of linear programs with linear complementarity constraints. SIAM Journal on Optimization, 19(1): 445-471, doi: http://dx.doi.org/10.1137/07068463x.

(18) Büeler, B., Enge, A., Fukuda, K. (2000). Exact volume computation for polytopes: A practical study. In Polytopes - Combinatorics and computation, (pp. 131-154). Basel: Birkhäuser. doi: http://dx.doi.org/10.1007/978-3-0348-8438-9_6.

(19) Büeler, B., Enge, A. (2003). VINCI version 1.o.5, Computing volumes of convex polytopes. Palaiseau-France: Laboratoire d'Informatique École polytechnique (bajo licencia GNU-GPL).

(20) Rubinstein, R.Y., Kroese, D.P. (2007). Simulation and the Monte Carlo method. Wiley. doi: http://dx.doi. org/10.1002/9780470230381. 